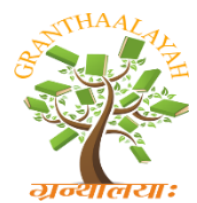

\author{
INTERNATIONAL JOURNAL OF RESEARCH \\ GRANTHAALAYAH \\ A knowledge Repository
}

Science

\title{
DETERMINATION OF ELECTRIC FIELD IN TERMS OF CURRENT AND CHARGE BY THE CONTINUITY EQUATION APPROACH
}

\author{
Pitri Bhakta Adhikari *1 \\ ${ }^{* 1}$ Department of Physics, Tri - Chandra Campus, TU, Nepal
}

\begin{abstract}
To determine an expression for the electric field in terms of current density and charge density is used the continuity equation approach. In this approach, the expression of electric field using scalar and vector potential relates the charge density and current density. The major consequence of these equations is that they visualize how varying electric fields propagate at the speed of light. In Maxwell's electrodynamics, formulated as it is in terms of charge and current densities, a point charge must be regarded as the limit of an extended charge, when the size goes to zero. Hence, the total electric field at the point $\mathrm{P}$ is

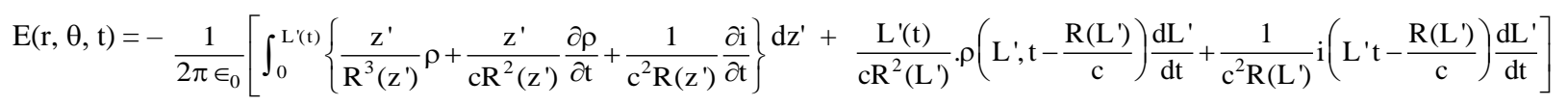

Keywords: Electric Fields; Current Density; Charge Density; Continuity Equation Approach.

Cite This Article: Pitri Bhakta Adhikari (2019). "Determination of Electric Field in Terms of Current and Charge by the Continuity Equation Approach." International Journal of Research Granthaalayah, 7(4), 162-170. https://doi.org/10.29121/granthaalayah.v7.i4.2019.885.
\end{abstract}

\section{Introduction}

The electrostatic discharge occurs between electrically charged regions may be between the two clouds, or cloud and air, or between a cloud and a ground. This discharge is called lightning discharge [1] - [5]. If there are a sufficiently charges in these regions which produce the high electric potential between them. If the charges in the cloud sufficiently large over the surface of the earth, an equal electric charge of opposite polarity is induced on the earth surface. The greater the accumulation of charges, higher will be the electric field produced. The electric field expression in the form of electro static, induction, and radiation fields were obtained in various papers. These components are very important for the lightning discharge [6] - [16]. There are so many approach used to determine the electric field. In the continuity equation approach, the expression of electric field using scalar and vector potential relates the charge density and current density. To determine an expression for the electric field in terms of current density and charge density is used in this paper. 


\section{Theory and Discussion}

To determine an expression for the electric field in terms of current density and charge density is used the continuity equation approach. In this approach, the expression of electric field using scalar and vector potential relates the charge density and current locally. The relation between the charge density and current at the retarded time is

$$
\frac{\partial \rho\left(z^{\prime}, t_{r}\right)}{\partial t}=-\left.\frac{\partial i\left(z^{\prime}, t_{r}\right)}{\partial z^{\prime}}\right|_{t_{r}=\text { constant }}
$$

Where, $t_{r}$ is the retarded time which is equal to $t_{r}=t-\frac{R\left(z^{\prime}\right)}{c}$.

In the right hand side of this equation, the partial differentiation of the current with source coordinate (z') keeping at the retarded time constant which is balanced with the rate of change of charge density.

Let us consider, in the figure 1, the return stroke starting from the point $\mathrm{A}$ on the ground in which $\mathrm{Z}^{\prime}=0$, then the charge is

$$
Q\left(t-\frac{r}{c}\right)=-\int_{r / c}^{t} i\left(0,0-\frac{r}{c}\right) d \tau
$$

The scalar potential due to the whole lightning channel is,

$$
\phi(r, t)=\quad \frac{1}{4 \pi \in_{0}} \frac{Q(t-r / c)}{r}+\frac{1}{4 \pi \in_{0}} \int_{0}^{L^{\prime}(t)} \rho\left(z^{\prime}, t-\frac{R\left(z^{\prime}\right)}{c}\right) d z^{\prime}
$$

The negative gradient of the scalar potential by using the spherical coordinate system is given by [17] - [20]

$$
\begin{aligned}
-\nabla \phi=-\frac{1}{4 \pi \epsilon_{0}}[ & \hat{\mathrm{r}} \frac{\partial}{\partial \mathrm{r}} \frac{\mathrm{Q}(\mathrm{t}-\mathrm{r} / \mathrm{c})}{\mathrm{r}}+\hat{\mathrm{r}} \frac{\partial}{\partial \mathrm{r}} \int_{0}^{\mathrm{L}^{\prime}(\mathrm{t})} \frac{1}{\mathrm{R}\left(\mathrm{z}^{\prime}\right)} \rho\left(\mathrm{z}^{\prime}, \mathrm{t}-\frac{\mathrm{R}\left(\mathrm{z}^{\prime}\right)}{\mathrm{c}}\right) \mathrm{d} \mathrm{z}^{\prime} \\
& \left.+\hat{\theta} \frac{1}{\mathrm{r}} \int_{0}^{\mathrm{L}^{\prime}(\mathrm{t})} \frac{\partial}{\partial \theta} \frac{\rho\left(\mathrm{z}^{\prime}, \mathrm{t}-\frac{\mathrm{R}\left(\mathrm{z}^{\prime}\right)}{\mathrm{c}}\right)}{\mathrm{R}\left(\mathrm{z}^{\prime}\right)} \mathrm{dz} \mathrm{z}^{\prime}\right]
\end{aligned}
$$




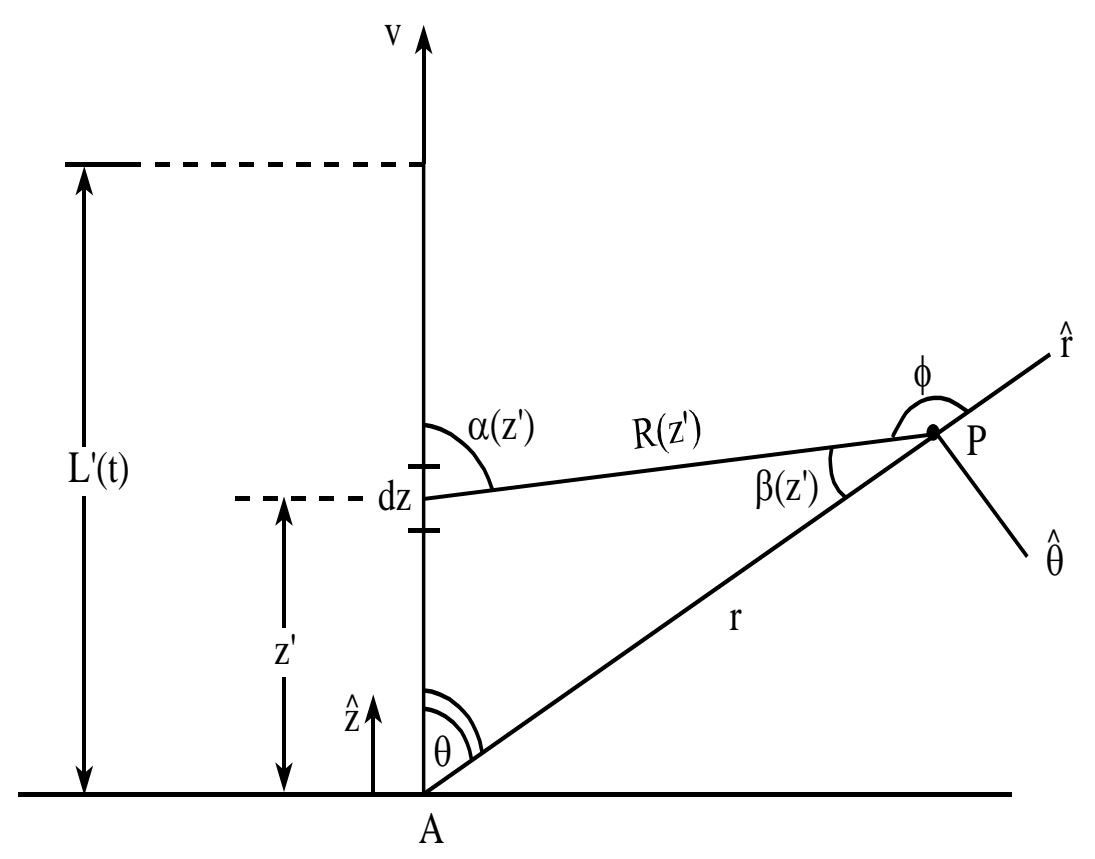

Figure 1: Geometry of the problem to determine the electric Field by the continuity equation approach.

From the figure, we can write,

$$
\begin{aligned}
\mathrm{R}^{2}\left(\mathrm{z}^{\prime}\right) & =\mathrm{r}^{2}+\mathrm{z}^{\prime 2}-2 r \mathrm{z}^{\prime} \cos \theta \\
\text { Or, } \mathrm{R}\left(\mathrm{z}^{\prime}\right)= & \sqrt{\mathrm{r}^{2}+\mathrm{z}^{\prime 2}-2 \mathrm{rz}^{\prime} \cos \theta}
\end{aligned}
$$

Here, the $\mathrm{R}\left(\mathrm{z}^{\prime}\right)$ is the function of both $\mathrm{r}$ and $\theta$, so on the partial differentiation,

$$
\begin{array}{ll}
\frac{\partial \mathrm{R}\left(\mathrm{z}^{\prime}\right)}{\partial \theta}= & \frac{1}{2}\left(\mathrm{r}^{2}+\mathrm{z}^{\prime 2}-2 \mathrm{rz} \mathrm{z}^{\prime} \cos \theta\right)^{-1 / 2} \cdot 2 \mathrm{rz} \mathrm{z}^{\prime} \sin \theta \\
\frac{\partial \mathrm{R}\left(\mathrm{z}^{\prime}\right)}{\partial \theta}= & \frac{\mathrm{rz} \mathrm{z}^{\prime} \sin \theta}{\mathrm{R}\left(\mathrm{z}^{\prime}\right)}
\end{array}
$$

Again, $\frac{\partial \mathrm{R}\left(\mathrm{z}^{\prime}\right)}{\partial \mathrm{r}}=\frac{1}{2}\left(\mathrm{r}^{2}+\mathrm{z}^{\prime 2}-2 \mathrm{rz} \mathrm{z}^{\prime} \cos \theta\right)^{-1 / 2} \cdot\left(2 \mathrm{r}-2 \mathrm{z}^{\prime} \cos \theta\right)$

$$
=\frac{r-z^{\prime} \cos \theta}{R\left(z^{\prime}\right)}
$$

Now, $\frac{\partial \rho}{\partial r}=\frac{\partial \rho}{\partial R} \cdot \frac{\partial R}{\partial r}=-\frac{1}{c} \frac{\partial \rho}{\partial t} \cdot \frac{r-z^{\prime} \cos \theta}{R\left(z^{\prime}\right)}$

$$
\text { and } \frac{\partial \rho}{\partial \theta}=\frac{\partial \rho}{\partial \mathrm{R}} \cdot \frac{\partial \mathrm{R}}{\partial \theta}=-\frac{1}{\mathrm{c}} \frac{\partial \rho}{\partial \mathrm{t}} \cdot \frac{\mathrm{rz} \mathrm{z}^{\prime} \sin \theta}{\mathrm{R}\left(\mathrm{z}^{\prime}\right)}
$$


Where $\rho=\rho\left(z^{\prime}, t-\frac{R\left(z^{\prime}\right)}{c}\right)$

$$
\frac{\partial \rho}{\partial t}=\frac{\partial \rho}{\partial z^{\prime}} \text { but } \frac{\partial \rho}{\partial R}=-\frac{1}{c} \frac{\partial \rho}{\partial t}
$$

On substituting all these values in the above equation

$$
\begin{aligned}
& -\nabla \phi=-\frac{1}{4 \pi \epsilon_{0}}\left[\hat{\mathrm{r}} \frac{\partial}{\partial \mathrm{r}} \frac{\mathrm{Q}(\mathrm{t}-\mathrm{r} / \mathrm{c})}{\mathrm{r}}+\hat{\mathrm{r}} \frac{\partial}{\partial \mathrm{r}} \int_{0}^{\mathrm{L}^{\prime}(\mathrm{t})} \frac{1}{\mathrm{R}\left(\mathrm{z}^{\prime}\right)} \rho\left(\mathrm{z}^{\prime}, \mathrm{t}-\frac{\mathrm{R}\left(\mathrm{z}^{\prime}\right)}{\mathrm{c}}\right) \mathrm{dz} \mathrm{z}^{\prime}\right. \\
& \left.-\hat{\theta} \frac{1}{\mathrm{r}} \int_{0}^{\mathrm{L}^{\prime}(\mathrm{t})} \frac{\partial}{\partial \theta} \frac{\rho\left(\mathrm{z}^{\prime}, \mathrm{t}-\frac{\mathrm{R}\left(\mathrm{z}^{\prime}\right)}{\mathrm{c}}\right)}{\mathrm{R}\left(\mathrm{z}^{\prime}\right)} \mathrm{dz} \mathrm{z}^{\prime}\right] \\
& \text { or, } \quad-4 \pi \in_{0} \nabla \phi=-\hat{\mathrm{r}} \frac{\partial}{\partial \mathrm{r}} \frac{\mathrm{Q}\left(\mathrm{t}-\frac{\mathrm{r}}{\mathrm{c}}\right)}{\mathrm{r}}-\hat{\mathrm{r}} \int_{0}^{\mathrm{L}^{\prime}(\mathrm{t})}\left[\frac{\mathrm{d} \rho\left(\mathrm{z}^{\prime}, \mathrm{t}-\frac{\mathrm{R}\left(\mathrm{z}^{\prime}\right)}{\mathrm{c}}\right)}{\partial \mathrm{r}} \cdot \frac{1}{\mathrm{R}\left(\mathrm{z}^{\prime}\right)}+\rho\left(\mathrm{z}^{\prime}, \mathrm{t}-\frac{\mathrm{R}\left(\mathrm{z}^{\prime}\right)}{\mathrm{c}}\right)\right. \\
& \left.-\frac{1}{\mathrm{R}^{2}\left(\mathrm{z}^{\prime}\right)} \cdot \frac{\partial \mathrm{R}}{\partial \mathrm{r}}\right] \mathrm{dz} \mathrm{z}^{\prime}-\frac{\hat{\theta}}{\mathrm{r}} \int_{0}^{\mathrm{L}^{\prime}(\mathrm{t})}\left[\frac{\partial \rho\left(\mathrm{z}^{\prime}, \mathrm{t}-\frac{\mathrm{R}\left(\mathrm{z}^{\prime}\right)}{\mathrm{c}}\right)}{\mathrm{d} \theta} \frac{1}{\mathrm{R}\left(\mathrm{z}^{\prime}\right)}+\left(-\frac{1}{\mathrm{R}^{2}\left(\mathrm{z}^{\prime}\right)}\right) \frac{\partial \mathrm{R}}{\partial \theta} \cdot \rho\right] \mathrm{dz} \\
& -\left[\frac{\hat{r} \rho\left(L^{\prime}\right)}{\mathrm{rR}\left(\mathrm{L}^{\prime}\right)} \frac{\mathrm{dL}}{\mathrm{dr}}\right]-\left[\frac{\hat{\theta} \rho\left(\mathrm{L}^{\prime}\right)}{\mathrm{rR}\left(\mathrm{L}^{\prime}\right)} \frac{\mathrm{dL}}{\mathrm{d} \theta}\right] \\
& \text { or, } \quad-4 \pi \in_{0} \nabla \phi=\quad \hat{r} \int_{0}^{L^{\prime}(t)}\left[\frac{r-z^{\prime} \cos \theta}{R^{3}(z)} \rho+\frac{r-z^{\prime} \cos \theta}{c^{2}\left(z^{\prime}\right)} \frac{\partial \rho}{\partial t}\right] d z^{\prime}+\hat{\theta} \int_{0}^{L^{\prime}(t)}\left[\frac{z^{\prime} \sin \theta}{R^{3}(z)} \rho+\frac{z^{\prime} \sin \theta}{c^{2}\left(z^{\prime}\right)} \frac{\partial \rho}{\partial t}\right] \\
& \mathrm{dz} z^{\prime}-\hat{\mathrm{r}} \frac{\partial(\mathrm{Q} / \mathrm{r})}{\partial \mathrm{r}}-\hat{\mathrm{r}} \frac{\rho(\mathrm{L})}{\mathrm{R}(\mathrm{L})} \frac{\partial \mathrm{L}^{\prime}}{\partial \mathrm{r}}-\hat{\theta} \frac{\rho\left(\mathrm{L}^{\prime}\right)}{\mathrm{rR}\left(\mathrm{L}^{\prime}\right)} \frac{\partial \mathrm{L}^{\prime}}{\partial \theta} \\
& \text { Here, } \rho \equiv \rho\left(z^{\prime}, t-\frac{R\left(z^{\prime}\right)}{c}\right) \\
& \rho\left(L^{\prime}\right)=\quad \rho\left(L^{\prime}, t-\frac{R\left(L^{\prime}\right)}{c}\right)
\end{aligned}
$$

The vector potential at $P$ due to the whole retarded length $L^{\prime}(t)$ is

$$
\overline{\mathrm{A}}(\mathrm{r}, \mathrm{t})=\frac{\mu_{0}}{4 \pi} \int_{0}^{\mathrm{L}^{\prime}(\mathrm{t})} \frac{\mathrm{i}\left(\mathrm{z}^{\prime}, \mathrm{t} \frac{\mathrm{R}\left(\mathrm{z}^{\prime}\right)}{\mathrm{c}}\right)}{\mathrm{R}\left(\mathrm{z}^{\prime}\right)} d \mathrm{z}^{\prime} \mathrm{z}
$$


Now, the negative rate of change of vector potential is

$$
-\frac{\partial \mathrm{A}}{\partial \mathrm{t}}=\quad \frac{1}{4 \pi \epsilon_{0}}(-\hat{z}) \int_{0}^{\mathrm{L}^{\prime}(\mathrm{t})}\left[\frac{1}{\mathrm{c}^{2} \mathrm{R}\left(\mathrm{z}^{\prime}\right)} \frac{\partial \mathrm{i}\left(\mathrm{z}^{\prime}, \mathrm{t}-\frac{\mathrm{R}\left(\mathrm{z}^{\prime}\right)}{\mathrm{c}}\right)}{\partial \mathrm{t}} \mathrm{dz} \mathrm{z}^{\prime}-\hat{\mathrm{z}} \frac{\mathrm{i}\left(\mathrm{L}^{\prime}, \mathrm{t}-\frac{\mathrm{R}\left(\mathrm{L}^{\prime}\right)}{\mathrm{c}}\right)}{\mathrm{c}^{2} \mathrm{R}\left(\mathrm{L}^{\prime}\right)} \frac{\mathrm{dL^{ \prime }}}{\mathrm{dt}}\right]
$$

Where, $\hat{z}=\hat{r} \cos \theta-\hat{\theta} \sin \theta$

On combining these two equations, then we get the total electric field at the point $\mathrm{P}$.

$$
\begin{aligned}
& \mathrm{E}(\rho, \theta, \tau)=-\nabla \phi+\frac{\partial \mathrm{A}}{\partial \mathrm{t}} \\
& \therefore \mathrm{E}(\rho, \theta, \tau)=\frac{1}{4 \pi \epsilon_{0}}\left[\hat{\mathrm{r}} \int_{0}^{\mathrm{L}^{\prime}(\mathrm{t})}\left\{\frac{\mathrm{r}-\mathrm{z}^{\prime} \cos \theta}{\mathrm{R}^{3}\left(\mathrm{z}^{\prime}\right)} \rho\left(\mathrm{z}^{\prime}, \mathrm{t}-\frac{\mathrm{R}\left(\mathrm{z}^{\prime}\right)}{\mathrm{c}}\right)+\frac{\mathrm{r}-\mathrm{z}^{\prime} \cos \theta}{\mathrm{cR}^{2}\left(\mathrm{z}^{\prime}\right)} \frac{\partial \rho\left(\mathrm{z}^{\prime}, \mathrm{t}-\mathrm{R}\left(\mathrm{z}^{\prime}\right) / \mathrm{c}\right)}{\partial \mathrm{t}}\right\} \mathrm{d} \mathrm{z}^{\prime}\right. \\
& +\hat{\theta} \int_{0}^{L^{\prime}(t)}\left\{\frac{z \sin \theta}{R^{3}\left(z^{\prime}\right)} \rho\left(z^{\prime}, t-\frac{R\left(z^{\prime}\right)}{c}\right)+\frac{z \sin \theta}{c R^{2}\left(z^{\prime}\right)} \frac{\partial \rho\left(z^{\prime}, t-R\left(z^{\prime}\right) / c\right)}{\partial t}\right\} d z^{\prime} \\
& -\hat{\mathrm{r}} \frac{\partial}{\partial \mathrm{r}}\left\{\frac{\mathrm{Q}(\mathrm{t}-\mathrm{r} / \mathrm{c})}{\mathrm{r}}\right\}-\hat{\mathrm{r}} \frac{\rho\left(\mathrm{L}^{\prime}\right)}{\mathrm{R}\left(\mathrm{L}^{\prime}\right)} \frac{\partial \mathrm{L}^{\prime}}{\partial \mathrm{r}}-\frac{\theta \rho\left(\mathrm{L}^{\prime}\right)}{\mathrm{rR}\left(\mathrm{L}^{\prime}\right)} \frac{\partial \mathrm{L}^{\prime}}{\partial \theta}-(\hat{\mathrm{r}} \cos \theta-\hat{\theta} \sin \theta) \\
& {\left[\int_{0}^{L^{\prime}(t)} \frac{1}{c^{2} R\left(z^{\prime}\right)} \frac{\partial i\left(z^{\prime}, t-R\left(z^{\prime}\right) / c\right)}{\partial t} d z^{\prime}-(\hat{r} \cos \theta-\hat{\theta} \sin \theta) \frac{i\left(L^{\prime}, t-\frac{R\left(L^{\prime}\right)}{c}\right)}{c^{2} R\left(L^{\prime}\right)} \frac{\partial L^{\prime}}{\partial t}\right]}
\end{aligned}
$$

Hence, we get the total electric field in spherical polar coordinate is

$E(r, \theta, t)=\frac{1}{4 \pi \epsilon_{0}} \hat{r} \int_{0}^{L^{\prime}(t)}\left\{\frac{r-z^{\prime} \cos \theta}{R^{3}\left(z^{\prime}\right)} \rho\left(z^{\prime}, t-\frac{R\left(z^{\prime}\right)}{c}\right)+\frac{r-z^{\prime} \cos \theta}{c R^{2}\left(z^{\prime}\right)} \frac{\partial \rho\left(z^{\prime}, t-R\left(z^{\prime}\right) / c\right.}{\partial t}\right\} d z^{\prime}$

$$
\begin{aligned}
& +\hat{\theta} \int_{0}^{L^{\prime}(t)}\left\{\frac{z^{\prime} \sin \theta}{R^{3}\left(z^{\prime}\right)} \rho\left(z^{\prime}, t-\frac{R\left(z^{\prime}\right)}{c}\right)+\frac{z^{\prime} \sin \theta}{c^{2}\left(z^{\prime}\right)} \frac{\partial \rho\left(z^{\prime}, t-R\left(z^{\prime}\right) / c\right)}{\partial t}\right\} d z^{\prime} \\
& -\hat{r} \int_{0}^{L^{\prime}(t)} \frac{\cos \theta}{c^{2} R\left(z^{\prime}\right)} \frac{\partial i\left(z^{\prime}, t-\frac{R\left(z^{\prime}\right)}{c}\right)}{\partial t} d z^{\prime}+\hat{\theta} \int_{0}^{L^{\prime}(t)} \frac{\sin \theta}{c^{2} R\left(z^{\prime}\right)} \frac{\partial i\left(z^{\prime}, t-\frac{R\left(z^{\prime}\right)}{c}\right)}{\partial t} d^{\prime}
\end{aligned}
$$




$$
\begin{aligned}
& -\hat{r} \frac{\cos \theta i\left(L^{\prime} t,-\frac{R\left(L^{\prime}\right)}{c}\right)}{c^{2} R\left(L^{\prime}\right)} \frac{d L^{\prime}(t)}{d t}+\frac{\hat{\theta} \sin \theta i\left(L^{\prime}, t-\frac{R\left(L^{\prime}\right)}{c}\right)}{c^{2} R\left(L^{\prime}\right)} \frac{d L^{\prime}(t)}{d t} \\
& +\hat{r}\left[\frac{1}{r^{2}} Q\left(t-\frac{r}{c}\right)+\frac{1}{r c} \frac{\partial Q\left(t-\frac{r}{c}\right)}{\partial t}\right]+\hat{r} \frac{1}{c R\left(L^{\prime}\right)} r\left(L^{\prime}, t-\frac{R\left(L^{\prime}\right)}{c}\right) \frac{d L}{d t} \\
& +\hat{\theta} \frac{\sin \theta}{c^{2} R\left(L^{\prime}\right)} \rho\left(L^{\prime} t-\frac{R\left(L^{\prime}\right)}{c}\right) \frac{d L^{\prime}(t)}{d t}
\end{aligned}
$$

Where, $\frac{\partial}{\partial \mathrm{r}}\left(\frac{\mathrm{Q}}{\mathrm{r}}\right)=-\frac{1}{\mathrm{r}^{2}} \mathrm{Q}+\frac{1}{\mathrm{r}} \cdot \frac{\partial \mathrm{Q}}{\partial \mathrm{r}}=-\frac{1}{\mathrm{r}^{2}} \mathrm{Q}-\frac{1}{\mathrm{cr}} \frac{\partial \mathrm{Q}}{\partial \mathrm{t}}$

Similarly, $\frac{\partial \mathrm{L}^{\prime}}{\partial \mathrm{r}}=-\frac{1}{\mathrm{c}} \frac{\partial \mathrm{L}}{\partial \mathrm{t}}$

and $\frac{\partial \mathrm{L}^{\prime}}{\partial \theta}=\frac{\partial}{\partial \theta}\left[\frac{\mathrm{v}}{1-(\mathrm{v} / \mathrm{c}) \cos \theta}\left(\mathrm{t}-\frac{\mathrm{r}}{\mathrm{c}}\right)\right]$

$$
\begin{aligned}
& =\quad \mathrm{v} \cdot\left(\mathrm{t}-\frac{\mathrm{r}}{\mathrm{c}}\right) \cdot(-1)\left[1-\left(\frac{\mathrm{v}}{\mathrm{c}}\right) \cos \theta\right]^{-2} \cdot\left(\frac{\mathrm{v}}{\mathrm{c}} \sin \theta\right) \\
& =\quad-\frac{\mathrm{v}(\mathrm{t}-\mathrm{r} / \mathrm{c})}{\left[1-\left(\frac{\mathrm{v}}{\mathrm{c}}\right) \cos \theta\right]^{2}}\left(\frac{\mathrm{v}}{\mathrm{c}} \sin \theta\right)
\end{aligned}
$$

In this long equation for the return stroke field at the ground level, then, $\theta=90^{\circ}$. So $\sin \theta=1$ and $\cos \theta=0$. Similarly, $\hat{\theta}=-\mathrm{z}$ since, $\hat{z}=\hat{\mathrm{r}} \cos \theta-\hat{\theta} \sin \theta$, the unit vector $\hat{r}$ becomes only horizontal as shown in figure, pointing away from the channel. At $z^{\prime}=0$, a perfectly conducting plane i.e. the earth is introduced to simulate the effect.

By using image theory, the total electric field should be added up i.e. the same of the field given by the image channel carrying current in the same direction as the actual channel. Hence, the total electric field at the point $\mathrm{P}$ is

$$
\begin{aligned}
& \mathrm{E}(\mathrm{r}, \theta, \mathrm{t})=-\frac{1}{2 \pi \epsilon_{0}}\left[\int_{0}^{\mathrm{L}^{\prime}(\mathrm{t})}\left\{\frac{\mathrm{z}^{\prime}}{\mathrm{R}^{3}\left(\mathrm{z}^{\prime}\right)} \rho+\frac{\mathrm{z}^{\prime}}{\mathrm{cR}^{2}\left(\mathrm{z}^{\prime}\right)} \frac{\partial \rho}{\partial \mathrm{t}}+\frac{1}{\mathrm{c}^{2} \mathrm{R}\left(\mathrm{z}^{\prime}\right)} \frac{\partial \mathrm{i}}{\partial \mathrm{t}}\right\} \mathrm{dz}\right. \\
& \left.+\frac{L^{\prime}(t)}{c R^{2}\left(L^{\prime}\right)} \cdot \rho\left(L^{\prime}, t-\frac{R\left(L^{\prime}\right)}{c}\right) \frac{d L^{\prime}}{d t}+\frac{1}{c^{2} R\left(L^{\prime}\right)} i\left(L^{\prime} t-\frac{R\left(L^{\prime}\right)}{c}\right) \frac{d L^{\prime}}{d t}\right]
\end{aligned}
$$


The first three terms of this equation are similar to be corresponding terms of the expression of the determination of the electro-magnetic field in the Jefimenko equation. If there is no current in the wave front then the last two terms Vanishes and only we get the first three terms:

$$
\mathrm{E}(\mathrm{r}, \theta, \mathrm{t})=-\frac{1}{2 \pi \epsilon_{0}}\left[\int_{0}^{\mathrm{L}^{\prime}(\mathrm{t})}\left\{\frac{\mathrm{z}^{\prime}}{\mathrm{R}^{3}\left(\mathrm{z}^{\prime}\right)} \rho+\frac{\mathrm{z}^{\prime}}{\mathrm{cR}^{2}\left(\mathrm{z}^{\prime}\right)} \cdot \frac{\partial \rho}{\partial \mathrm{t}}+\frac{1}{\mathrm{c}^{2} \mathrm{R}\left(\mathrm{z}^{\prime}\right)} \frac{\partial \mathrm{i}}{\partial \mathrm{t}}\right\} \mathrm{dz}^{\prime}\right]
$$

\section{Conclusion}

The first term of this equation represent the electrostatic field which have $\mathrm{R}^{-3}$ term, the second term contain $\mathrm{c}^{-1} \mathrm{R}^{-2}$, which represents the induction field, and the last term represents the radiation field containing terms. $1 / \mathrm{c}^{2} \mathrm{R}\left(\mathrm{z}^{\prime}\right)$. The graph of these static field, induction field, radiation field and the total electric field are shown in figure 2. The individual effect of these components on the total electric field is evaluated. As the electrostatic component increases, the total electric field also increases proportionately as seen in the graph. Similarly the total electric field increases as the induction field increased but the effect of induction component is less when compared to that of the electrostatic field. In contrast, there is opposite relation between the radiation component and the total electric field which suggests that decrease in the radiation component increases the total electric field. Hence, we can conclude that the sum of the electrostatic component and the induction component of the electric field has relatively greater effect to the total electric field than the radiation components in the lightning discharge.
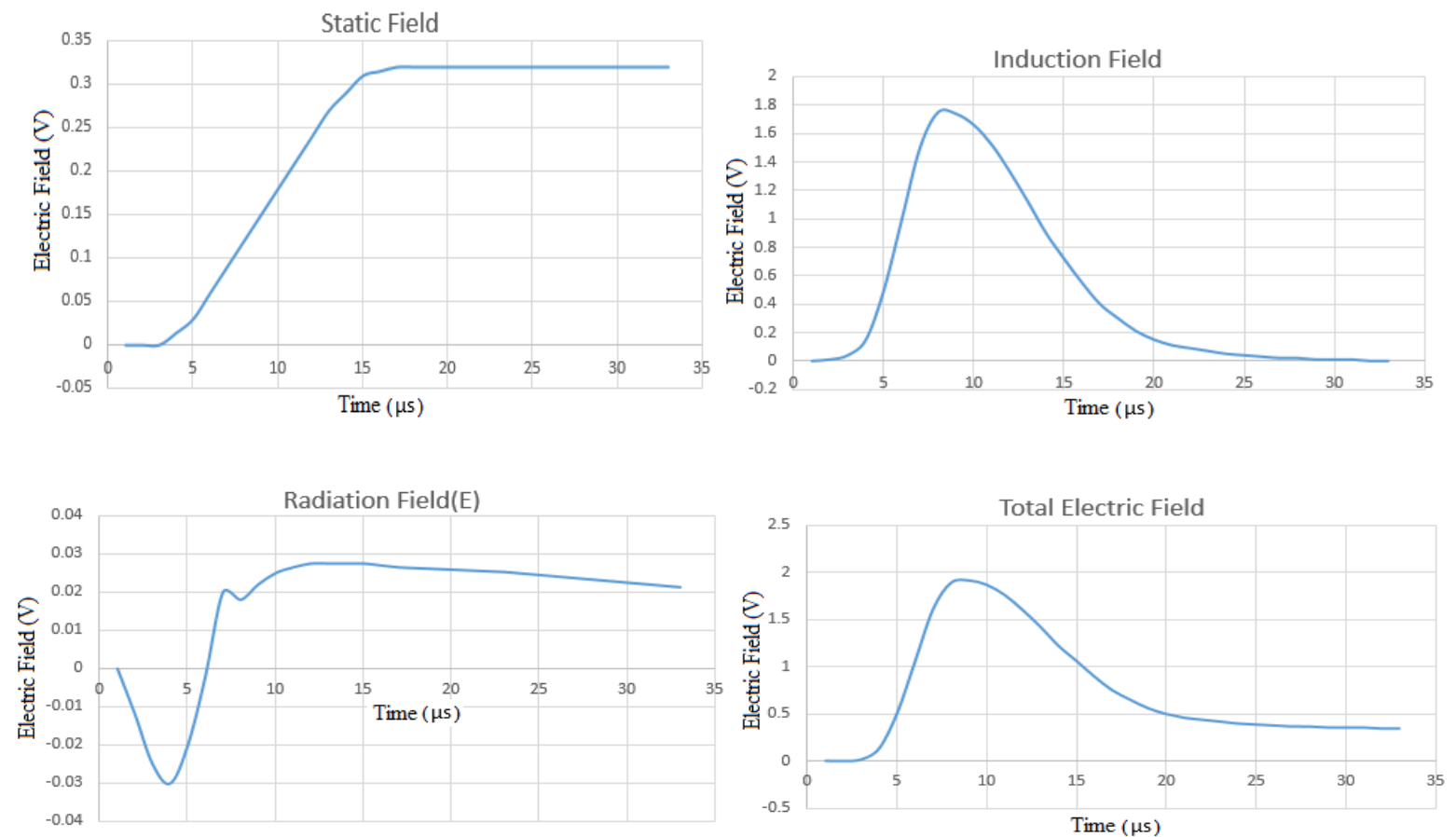

Figure 2: The three components of the electric field due to lightning and total electric field are given in the figure 2 . 


\section{Acknowledgement}

I would like to express our sincere gratitude to the International Science Programme (ISP), Uppsala University, Sweden, for providing the instruments necessary for this research in this project to measure the electric field. It would not have been possible to carry out this study without their help. Also, we would like to thank the Tri-chandra College, Amrit campus and Central Department of Physics Tribhuvan University for the research facility.

\section{References}

[1] Bazelyan, E. M., \& Raizer, Y. P. (2000). Lightning Physics and Lightning Protection. london: Institute of Physics Publishing.

[2] Adhikari, P. B., Sharma, S. R., \& Baral, K. N. (2016). Features of positive ground flashes observed in Kathmandu Nepal. Journal of Atmospheric and Solar-Terrestrial Physics, 145, 106113. homepage: www.elsevier.com/ locate/jastp

[3] Adhikari, P. B., Sharma, S. R., Baral, K. N., \& Rakov V.A. (2017). Unusual lightning electric field waveforms observed in Kathmandu, Nepal, and Uppsala, Sweden (2017); Journal of Atmospheric and Solar-Terrestrial Physics, 164, 172-184. homepage: www.elsevier.com/ locate/jastp

[4] Adhikari, P. B., Bhandari, B (2017). Computation of Electric Field from Lightning Discharges; International Journal of Scientific \& Engineering Research Volume 8, Issue 9, 147 ISSN 2229-5518.

[5] Adhikari, P.B. (2017). Features of ground flashes before and after the massive earthquake observed from Kathmandu, Nepal. Journal of Nepal Physical Society, Volume 4, Issue 1, 11-22, (2017), ISSN: 2392-473X.

[6] Thottappillil, R., Uman, M., \& Rakov, V. (1998). Treatment of retardation effects in calculating the radiated electromagnetic fields from the lightning discharge. Journal of Geophsical Research, 103 (D8), 9003-9013.

[7] Thottappillil, R., Rakov, V. A., \& Uman, M. A. (1997). Distribution of charge along the lightning channel: Relation to remote electric and magnetic fields and to return-stroke models. Journal of Geophysical Research, 102 (D6), 6987-7006.

[8] Thottappillil, R., Schoene, J., \& Uman, M. (2001). Return stroke transmission line model for stroke speed near and equal that of light. Geophysical Research Letters, 28, pp. 3593-3596.

[9] Chalmers, J. A. (1965). The so-called "induction" component in the electric field of a lightning discharge. Journal of Atmospheric and Solar-Terrestial Physics, 27 (9), 1027-1028.

[10] Shao, X. M. (2016). Generalization of the lightning electromagentic equations of Uman, Mclain, and Krieder based on Jefimenko equation, Journal of Geophysical Research, 121, 3363- 3371.

[11] Nickolaenko, A. P., \& Hayakawa, M. (1998). Electric fields produced by lightning discharge. Journal of Geophysical research: atmospheres, 103 (D14), 17175-17189.

[12] Chalmers, J. A. (1965). The so-called "induction" component in the electric field of a lightning discharge. Journal of Atmospheric and Terrestial Physics, 27 (9), 1027-1028.

[13] Thottappillil, R., \& Rakov, V. A. (2001). On different approaches to calculating lightning electric fields. Journal of GeoPhysical research, 106 (NO. D13), 14191-14205.

[14] Adhikari, P. B., (2017), analyzing the components of lightning electric field. GoldenGate Journal of Science \& Technology, 3, 22 - 26. ISSN 2505 -0656.

[15] Adhikari, P. B., (2018), Time Varying Electric and Magnetic Fields from Lightning Discharge. International Journal of Electrical and Electronic Science. Vol. 5, No. 2, pp. 50-55.

[16] Adhikari, P. B., Bhandari, B, (2018), Jefimenko Equations in Computation of Electromagnetic Fields for Lightning Discharges. International Journal of Scientific \& Engineering Research. Volume 9, Issue 6, pp 1678 - 1687, ISSN 2229-5518. 
[17] Griffith, D. (1999). Introduction to Electrodynamics. New Jersey: Prentice Hall, Upper Saddle River.

[18] Jefimenko, O. (1966). Electricity and Magnetism. New York: Appleton-Century-Crofts.

[19] Jefimenko, O. (1989). Electricity and Magnetism. Star City, West Virginia: Electret Scientific.

[20] Cooray, V. (2003). The Lightning Flash. The Institution of Electrical Engineers (IEE), London.

*Corresponding author.

E-mail address: pbadhikari09@ gmail.com 